\title{
DOI: 10.3901/JME.2020.16.054
}

\section{氢致 TA10 钛合金焊接接头断裂韧度演变研究*}

\author{
刘全明 ${ }^{1,2}$ 龙伟民 $^{1}{\text { 傅 } \text { 莉 }^{2} \text { 张朝晖 }^{3} \text { 张 雷 }}^{1}$ 宋晓国 ${ }^{4}$ \\ (1. 郑州机械研究所有限公司新型钎焊材料与技术国家重点实验室 郑州 450001; \\ 2. 西北工业大学凝固技术国家重点实验室 西安 710072;
}

3. 西安建筑科技大学冶金工程学院 西安 710055;

4. 哈尔滨工业大学先进焊接与连接国家重点实验室 哈尔滨 150001)

\begin{abstract}
摘要：钛合金焊接件在氢环境中服役面临着氢脆断裂风险，断裂韧度对钛合金损伤容限设计至关重要。研究充氢量对钛合金 焊接接头断裂韧度、断口形貌和断裂方式的影响, 揭示了固溶氢、氢化物导致焊接接头断裂韧度演变的微观机理。充氢 0 ～ $0.21 \mathrm{wt}$. \% CT 试样断裂韧度 $K_{Q}$ 值由 $39.6 \mathrm{MPa} \cdot \mathrm{m}^{1 / 2}$ 减小到 $22.1 \mathrm{MPa} \cdot \mathrm{m}^{1 / 2}$, 扩展区断口形貌由大量韧窝+局部少量脆性断裂 转为脆性断裂, 氢致断裂韧度损失和脆性断裂加剧。应力诱导氢扩散使固溶氢局部富集形成富氢气团, 气团存在内压剪切分 量使产生局部塑性变形所需外应力下降, 焊接接头表观屈服应力下降, 促进微裂纹在低 $K_{I}$ 下孕育。位错运动无法直接切过大 尺寸氢化物, 位错塞积在氢化物界面处, 致局部应力集中, 微裂纹在氢化物界面处孕育; 同时氢化物为软基体金属中的一种 脆性相, 应力集中致其产生高应变, 氢化物自身断裂, 微裂纹在氢化物内部孕育; 固溶氢、氢化物均致焊接接头加速开裂。
\end{abstract} 关键词: 钛合金; 充氢焊接接头; 断裂韧度; 断口形貌; 演变机理 中图分类号: TG146

\section{Fracture Toughness Evolution Induced by Hydrogen of TA10 Titanium Alloy Welded Joints}

\author{
LIU Quanming ${ }^{1,2} \quad$ LONG Weimin $^{1} \quad$ FU Li $^{2} \quad$ ZHANG Zhaohui ${ }^{3}$ ZHANG Lei ${ }^{1}$ \\ SONG Xiaoguo ${ }^{4}$
}

(1. State Key Laboratory of Advanced Brazing Filler Metals and Technology, Zhengzhou Research Institute of Mechanical Engineering Co., Ltd., Zhengzhou 450001;

2. State Key Laboratory of Solidification Processing, Northwestern Polytechnical University, Xi' an 710072;

3. School of Metallurgical Engineering, Xi'an University of Architecture and Technology, Xi'an 710055;

4. State Key Laboratory of Advanced Welding and Joining, Harbin Institute of Technology, Harbin 150001)

\begin{abstract}
Titanium alloy weldments in hydrogen environment are facing with the risk of hydrogen embrittlement, and the fracture toughness is very important for the damage tolerance design of titanium alloy. The effects of hydrogen content on fracture toughness, fracture morphology and fracture mode of titanium alloy welded joints were investigated. Micro-mechanisms of fracture toughness evolution induced by solid solution hydrogen and the hydride for welded joints were revealed. Fracture toughness $K_{Q}$ values of the hydrogenated $0-0.21 \mathrm{wt} . \% \mathrm{CT}$ specimens were decreased from $39.6 \mathrm{MPa} \cdot \mathrm{m}^{1 / 2}$ to $22.1 \mathrm{MPa} \cdot \mathrm{m}^{1 / 2}$, the fracture morphology of expansion zone was changed from a large number of dimples + a small amount of local brittle fracture to brittle intergranular fracture, and fracture toughness loss and brittle fracture induced by hydrogen for welded joints were increased. Hydrogen rich air masses were produced by local enrichment of solid solution hydrogen induced by stress-induced hydrogen diffusion. The existence of internal pressure shear component in hydrogen rich air masses resulted in the decrease of external stress required for local plastic deformation, the decrease of apparent yield stress and the promotion of microcracks inoculation at a lower $K_{I}$ level. The dislocation movement could not directly cut the large size of the hydride, and the dislocation plug was generated at the hydride interface, which caused local stress concentration, and the microcracks were incubated at the hydride interface. Besides, the hydride was a brittle phase in the soft
\end{abstract}

* 国家自然科学基金(U1904197)和河南省科技创新人才计划(204200510031)

资助项目。20200312 收到初稿, 20200422 收到修改稿 
matrix metal, which caused high strain due to stress concentration, the hydride itself broke, and the microcracks were incubated inside the hydride. The accelerated cracking of welded joints was caused by solid solution hydrogen and the hydride at once.

Key words: titanium alloy; hydrogenated welded joint; fracture toughness; fracture morphology; evolution mechanism

\section{0 前言}

钛合金兼有比强度大、耐腐蚀、高韧性、可焊 接和高疲劳裂纹扩展抗力等优良特性, 目前广泛应 用于航空、航天、深潜装备制造领域。加氢反应器、 航天器燃料箱、深潜器等大型装备制造大多采用高强 钛合金焊接结构, 结构件长期服役于氢环境中发生塑 性变形概率较低, 但由于钛与氢亲和力较强, 很难排 除其发生氢脆可能。氢脆大多在低于合金屈服强度下 突然断裂，存在巨大安全隐患，开展钛合金焊接结构 损伤容限设计尤为重要。伴随断裂力学理论在高端装 备设计制造领域中的应用程度提升, 作为材料阻止宏 观裂纹失稳扩展能力度量的断裂韧度逐步成为材料 能否满足结构设计的重要指标之一 ${ }^{[1-4]}$ 。平面应变断裂 㓞度表征了材料在含有裂纹体时的断裂抗力, 决定关 键结构件寿命, 被广泛用于工程材料抗断裂设计 ${ }^{[5]}$, 同时为评价材料损伤容限性能重要参考。前期钛合金 断裂韧度研究大多针对某一类钛合金 ${ }^{[6-11]}$ 或钛合金焊 接接头 ${ }^{[12-13]}$, 氢致钛合金焊接接头断裂韧度演变研究 明显缺乏, 结构件氢损伤数据并不完整, 氢脆失效控 制、预防和氢损伤评估、寿命预测研究更是无从谈起。 本文拟分析充氢量对 TA10(Ti-0.3Mo-0.8Ni)钛合金焊 接接头断裂韧度和断口形貌的影响, 观察焊接接头断 裂方式转变, 揭示固溶氢、氢化物对断裂韧度演变的 微观作用机理, 为后续钛合金结构件安全设计、选材 和剩余寿命预测奠定基础。

\section{1 试验方法}

通常断裂韧度指标由平面应变断裂韧度 $K_{I C}$ 表 征, 仅当试样尺寸满足平面应变状态或小范围屈服 力学条件时, 才能获得有效 $K_{I C}$ 值, 而在工程应用 中多数试样尺寸无法严格满足 $K_{I C}$ 值测试条件, 因 此 $K_{I C}$ 值可用测试条件值 $K_{Q}$ 值表示, $K_{Q}$ 值仅表示 裂纹失稳扩展阻力的相对大小。TA10 钛合金双面对 接板经手工铇极氩弧焊多道次焊成, 断裂韧度测试 采用紧凑拉伸(Compact tension, CT) 试样作为标准 试样, 将待充氢试样放置于 L2210II/2M 型管式氢处 理炉中, $750{ }^{\circ} \mathrm{C}$ 恒温下保温 $2 \mathrm{~h}$, 炉冷至 $170{ }^{\circ} \mathrm{C}$ 以 下, 控制充氢系统中 $\mathrm{H}_{2}$ 的平衡分压, 用精度为 $0.01 \mathrm{mg}$ 的电子天平测量试样充氢前后质量 ${ }^{[14]}$ 得到
目标充氢量 $(0.05 \mathrm{wt} . \% 、 0.12 \mathrm{wt} . \% 、 0.21 \mathrm{wt} . \%)$ 。CT 试样在 INSTRON 8801 型疲劳试验机上按 GB T21143-2014 金属材料准静态断裂韧度的统一试验 方法 ${ }^{[15]}$ 规定完成测试。图 1 为 CT 试样取样示意图, 钛合金氩弧焊板除去焊缝加厚高, 沿 $\mathrm{T}-\mathrm{L}$ 方向( $\mathrm{T}$ 为 断裂平面法线方向, $\mathrm{L}$ 同焊接方向平行)切取 CT 试样, 试样开缺口位置在焊缝区。CT 试样厚度约为 $8.5 \mathrm{~mm}$, 图 2 为 CT 试样几何形状与尺寸。预制裂纹由试验机 自带程序 “Fatigue Crack Propagation” 控制, 采用正 弦波加载, 应力比 $R=0.1$, 频率为 $20 \mathrm{~Hz}$, 应力强度 因子速率 $K=15 \mathrm{MPa} \mathrm{m}{ }^{1 / 2} \mathrm{~s}^{-1}$, 预制裂纹长度为 $1.8 \mathrm{~mm}$, 达到预设长度后, 再转至试验机自带程序 “KIC Test Control”, 采用恒载速率 $V=1.0 \mathrm{~mm} / \mathrm{min}$ 将 CT 试样拉 至最终断裂, 由计算得到所需 $K_{Q}$ 值。

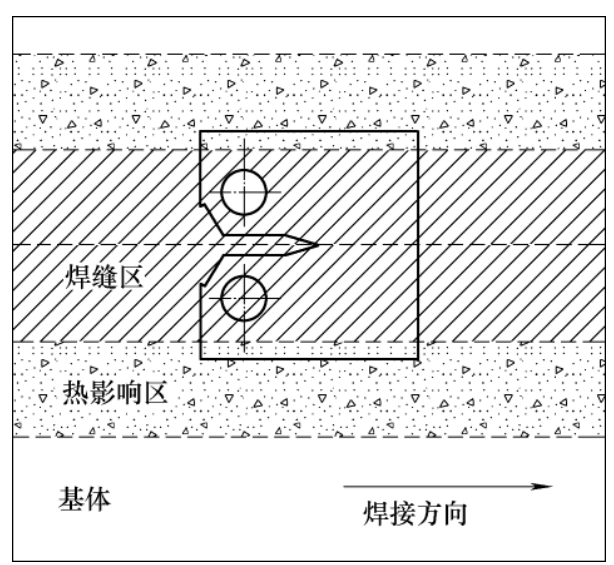

图 1 CT 试样取样示意图

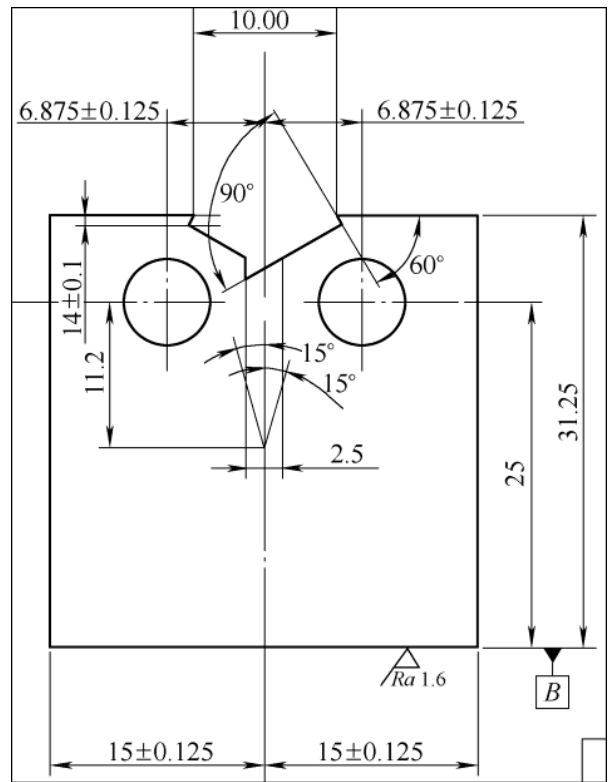

图 2 CT 试样几何形状与尺寸 


\section{2 断裂韧度演变}

钛合金断裂韧度强烈依赖于显微组织, 作者前期 研究 ${ }^{[16-17]}$ 表明充氢 $0.05 \mathrm{wt}$.\% 焊接接头组织中未析出氢 化物, 而充氢 $0.12 \mathrm{wt} . \%$ 和 $0.21 \mathrm{wt}$.\% 组织中析出多种氢 化物, 致显微组织明显改变。表 1 为不同充氢量 CT 试样断裂韧度 $K_{Q}$ 值测试结果。充氢 $0.05 \mathrm{wt} . \% \mathrm{CT}$ 试样 断裂韧度 $K_{Q}$ 值为 $39.6 \mathrm{MPa} \cdot \mathrm{m}^{1 / 2}$, 充氢 $0.12 \mathrm{wt}$ \% 和 $0.21 \mathrm{wt} . \%$ 时, $K_{Q}$ 值分别减小到 26.7 和 $22.1 \mathrm{MPa} \cdot \mathrm{m}^{1 / 2}$, 断裂韧度损失最高达 $44.19 \%$ 。充氢 $0 \sim 0.21 \mathrm{wt}$ \% 过程, 断裂㓞度随充氢量增加而逐步下降。

表 1 不同充氢量钛合金焊接接头断裂韧度 $K_{Q}$ 试验结果

\begin{tabular}{lccccc}
\hline 编号 & $\begin{array}{c}\text { 宽度 } \\
W / \mathrm{mm}\end{array}$ & $\begin{array}{c}\text { 厚度 } \\
B / \mathrm{mm}\end{array}$ & $\begin{array}{c}\text { 裂纹长度 } \\
a / \mathrm{mm}\end{array}$ & $\begin{array}{c}\text { 屈服强度 } \\
\sigma_{\rho 0.2} / \mathrm{MPa}\end{array}$ & $\begin{array}{c}\text { 断裂韧度 } \\
K_{Q} /\left(\mathrm{MPa} \cdot \mathrm{m}^{1 / 2}\right)\end{array}$ \\
\hline $\mathrm{CT}-1$ & 24.60 & 8.5 & 11.537 & 356 & 39.6 \\
$\mathrm{CT}-2$ & 24.54 & 8.5 & 11.530 & 379 & 26.7 \\
$\mathrm{CT}-3$ & 24.51 & 8.5 & 11.807 & 446 & 22.1 \\
\hline
\end{tabular}

\section{3 断口形貌}

试验主裂纹为典型张开型裂纹, 其断裂过程分为 裂纹萌生、扩展和瞬时断裂三个阶段, 对应断口形貌 依次为裂纹源(预裂)、扩展和快速断裂区。图 3 为不

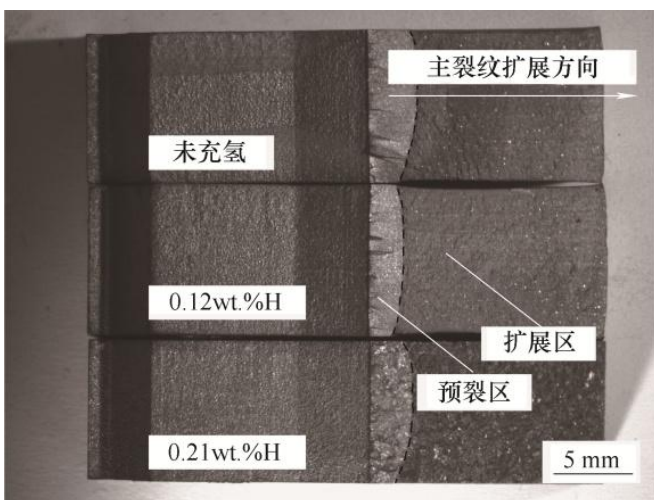

(a) 宏观断口形貌

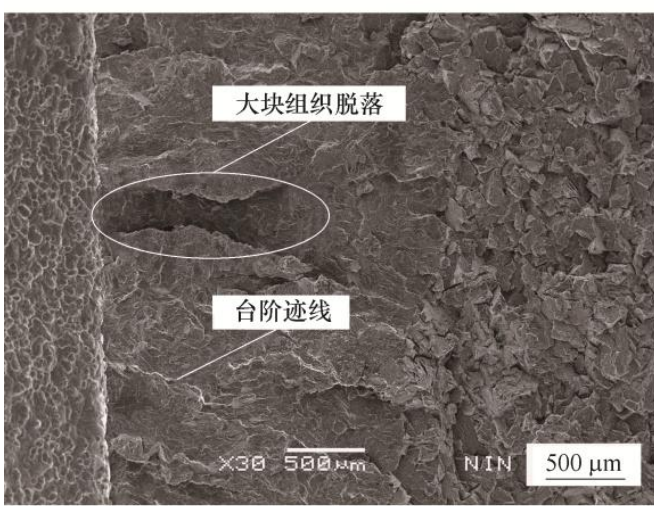

(b) 充氢 $0.21 w t . \%$ 试样低倍断口

图 3 CT 试样低倍断口形貌
同充氢量 CT 试样低倍断口形貌。图 3a 中虚线左侧为 预裂区，右侧为扩展区，主裂纹从左向右扩展。图 $3 \mathrm{~b}$ 所示, 预裂区断口为带有一定弧度的光滑扇形面, 微 裂纹一般源自扇柄处, 微裂纹扩展过程伴随尺寸增加、 多裂纹汇集，择优选出主裂纹。宏观断口上诸多可视 裂纹并非处在同一平面上，判定预裂区的典型形貌特 征为断口是否存在和主裂纹扩展方向相平行的台阶迹 线。进入扩展区, 断口形貌颜色灰暗, 粗䊁度更高; 快速断裂区并不明显。随充氢量增加, 预裂区和扩展区 分界线越发模糊不清，预裂区有大块组织脱落现象，扩 展区表面粗糙度降低，充氢致焊接接头脆性断裂加剧。

图 4 和图 5 分别为不同充氢量 CT 试样断口预 裂区和扩展区形貌。充氢 $0.05 \mathrm{wt}$.\% 时，预裂区断口 呈典型类解理形貌, 图 $4 \mathrm{a}$ 为类解理羽毛花样; 充氢 0.12 wt. \% 和 0.21 wt. \% 时，断口仍为类解理形貌，但 局部存在少量早期疲劳条带，疲劳条带略有弯曲并 大致平行排列，疲劳条带区随着充氢量增加略有扩 大(图 4b、4c)。充氢 $0.05 w t . \% 、 0.12 w t . \%$ 和 $0.21 w t . \%$ 时, 扩展区断口形貌依次为大量韧窝聚集+局部脆性 断裂(图 5a)、脆性沿晶断裂为主+局部少量韧窝聚集 (图 5b)和基本转为脆性沿晶断裂(图 5c)形貌。随着 充氢量增加, 㓞窝数量明显减少, 焊接接头塑性急 剧下降, 断裂方式由韧性断裂转为脆性断裂。

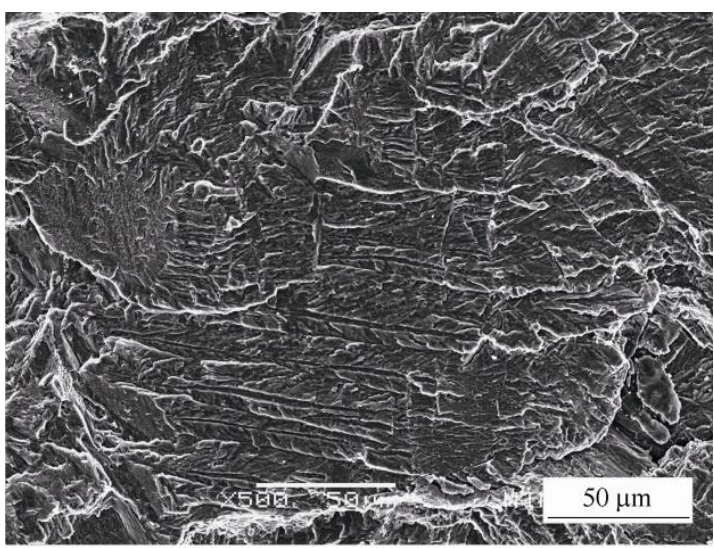

(a) $0.05 \mathrm{wt} . \% \mathrm{H}$

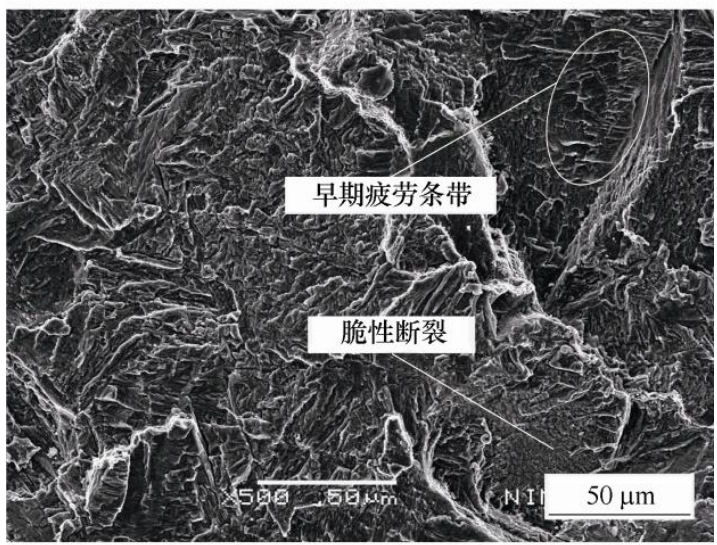

(b) $0.12 \mathrm{wt} . \% \mathrm{H}$ 


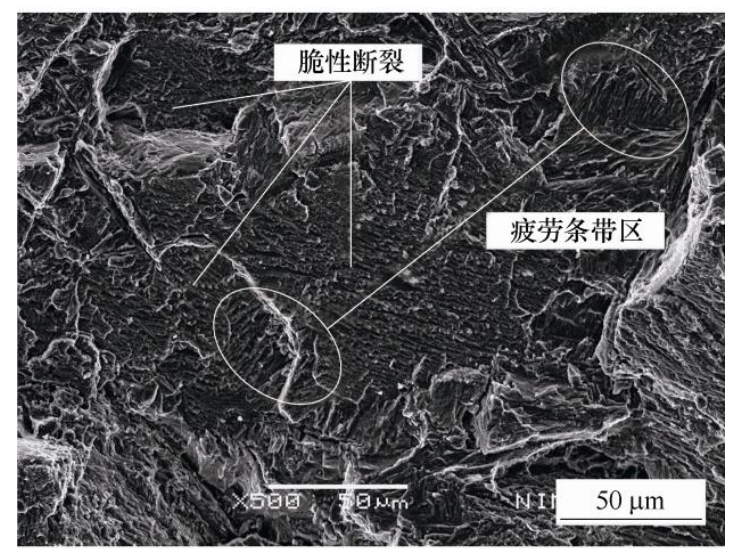

图 4 不同充氢量 CT 试样断口预裂区形貌

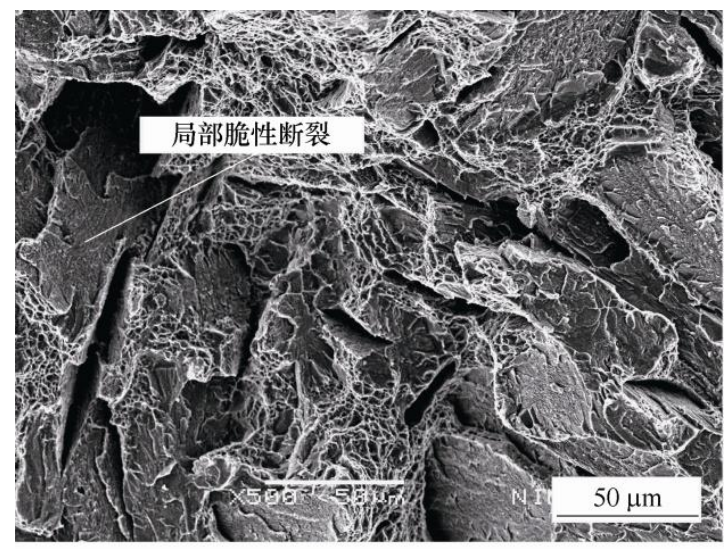

(a) $0.05 \mathrm{wt} . \% \mathrm{H}$

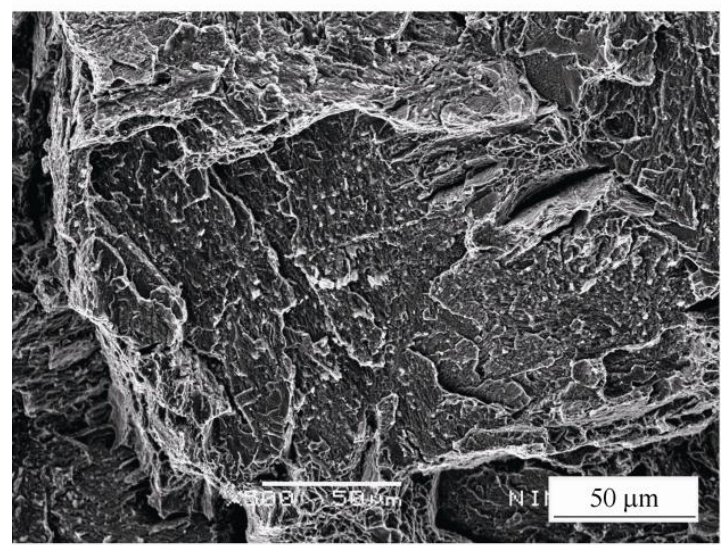

(b) $0.12 \mathrm{wt} . \% \mathrm{H}$

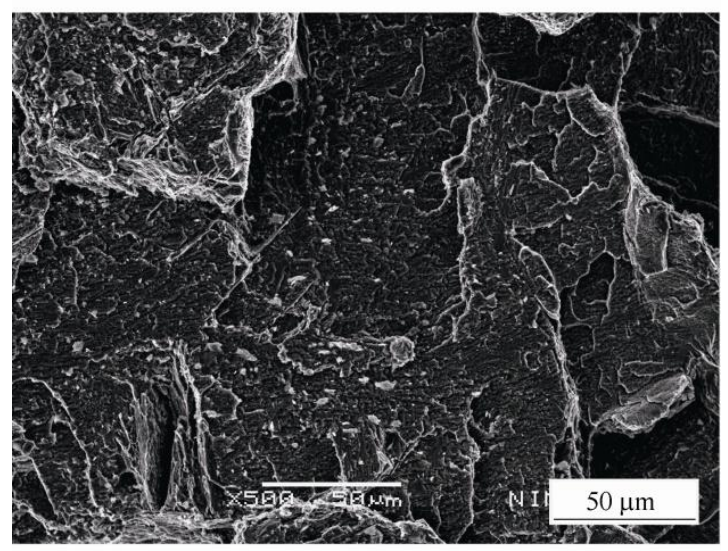

(c) $0.21 \mathrm{wt} . \% \mathrm{H}$

图 5 不同充氢量 $\mathrm{CT}$ 试样断口扩展区形貌

\section{4 氢致断裂韧度演变机理}

低氢焊接接头中含有固溶氢, $\mathrm{CT}$ 试样在循环加 载条件下产生应力梯度, 应力诱导氢扩散致固溶氢 发生局部富集; 氢致弱键、氢致表面能下降和氢致 局部塑性变形理论研究表明: 固溶氢致焊接接头加 速开裂。高氢焊接接头中析出氢化物, 在小应力作 用下, 氢化物自身断裂或沿基体界面开裂, 促进微 裂纹形成, 加速焊接接头开裂。断裂韧度表征材料 抵抗裂纹扩展能力, 氢致焊接接头断裂韧度下降表 观原因为氢加速焊接接头开裂, 其本质为氢致开裂 所需应力强度因子 $K_{I}$ 持续下降。本节借助氢致表观 屈服强度下降和微裂纹孕育位错塞积理论, 探讨固 溶氢、氢化物致焊接接头断裂韧度演变微观机理。

\section{1 氢致表观屈服强度下降理论}

氢致局部塑性变形理论 ${ }^{[18]}$ 研究表明: 任何断裂 过程均为局部塑性变形发展至临界状态的必然结果。 高氢焊接接头断口呈脆性沿晶断裂形貌, 微裂纹萌 生、扩展以位错运动(局部塑性变形)为先决条件, 沿晶裂纹扩展与局部塑性变形关系密切。微裂纹扩 展阻力主要源自裂纹扩展前端塑性变形功 $U_{P}$, 如式 (1) 所示 ${ }^{[19]}$, 临界应力场强度因子 $K_{I C}^{*}$ 与 $U_{P}^{1 / 2}$ 呈正比, 局部塑性变形范围和程度越小, $U_{P}$ 越小, 则 $K_{I C}^{*}$ 越 小。在氢致开裂过程, 应力强度因子 $K_{I}$ 较高时, 断 口为韧窝断裂; $K_{I}$ 较低时, 断口转为准解理或沿晶 断裂。氢致焊接接头氢脆敏感性提升, 微裂纹萌生 所需应力强度因子 $K_{I}$ 持续下降, 若 $K_{I}$ 明显下降, 断口由韧性转为脆性断裂。断口呈韧窝或脆性断裂, 主要区别在于开裂前局部塑性变形范围和程度存在 显著差异, 致开裂所需应力强度因子 $K_{I}$ 有所不同。

$$
K_{I C}^{*}=\left[\frac{E}{1-v^{2}}\left(U_{P}+2 \Gamma\right)\right]^{\frac{1}{2}}=\left(\frac{E}{1-v^{2}}\right)^{\frac{1}{2}} U_{P}^{\frac{1}{2}}
$$

式中, $K_{I C}^{*}$ 为临界应力场强度因子, $E$ 为弹性模量, $v$ 为泊松比, $U_{P}$ 为塑性变形功, $\Gamma$ 为表面能。

充氢 $0.05 \mathrm{wt} . \% \mathrm{CT}$ 试样中, 氢主要以固溶氢形 式存在, 预制裂纹时产生应力梯度, 应力诱导氢扩 散致裂纹尖端处氢局部富集形成富氢气团。氢气团 内压剪切分量 $\tau_{H}$ 和外应力场剪切分量 $\tau_{C}^{*}$ 致裂纹尖 端局部区域位错增殖、运动, 若两剪切分量之和大 于位错大规模增殖、运动所需克服的阻力, 尖端区 域产生局部塑性变形, 发展至临界状态时导致 CT 试样断裂。氢气团内压剪切分量始终存在, 产生局 部塑性变形所需外应力势必下降, 焊接接头表观屈 服应力降低, 其推导过程如下。 
(1) 如图 6 所示, 假设 CT 试样中心位置贯穿一 条长度为 $2 a$ 的裂纹, 均匀拉应力 $\sigma$ 作用下裂纹张开, 裂纹尖端区域各点(坐标 $r 、 \theta$ ) 应力分量如式(2)所示 ${ }^{[20]}$

$$
\left\{\begin{array}{l}
\sigma_{x x}=\frac{K_{I}}{\sqrt{2 \pi r}} \cos \frac{\theta}{2}\left(1-\sin \frac{\theta}{2} \sin \frac{3 \theta}{2}\right) \\
\sigma_{y y}=\frac{K_{I}}{\sqrt{2 \pi r}} \cos \frac{\theta}{2}\left(1+\sin \frac{\theta}{2} \sin \frac{3 \theta}{2}\right) \\
\tau_{x y}=\frac{K_{I}}{\sqrt{2 \pi r}} \sin \frac{\theta}{2} \cos \frac{\theta}{2} \cos \frac{3 \theta}{2} \\
\sigma_{z}= \begin{cases}\frac{\sigma \sqrt{\pi a}}{\sqrt{2 \pi r}} v \cos \frac{\theta}{2} & \text { 平面应变 } \\
0 & \text { 平面应力 }\end{cases}
\end{array}\right.
$$

（2）若氢致微裂纹沿着和其延长线呈 $\beta= \pm \pi / 4$ 方向扩展, 并将坐标轴转动 $\beta=\pi / 4$ 后, 新坐标系 中切应力 $\tau$ 为局部塑性变形推动力, 表达式为 式 (3) ${ }^{[19]}$, 将 $\beta=\pi / 4$ 和式(2)代入式(4)中, 注意式(2) 中 $\theta=\pi / 4$, 为式(5)

$$
\begin{gathered}
\tau=\frac{1}{2}\left(\sigma_{y y}-\sigma_{x x}\right) \sin 2 \beta+\tau_{x y} \cos 2 \beta \\
\tau=\frac{1}{2}\left(\sigma_{y y}-\sigma_{x x}\right) \\
\tau=0.328 \frac{K_{I}^{*}}{\sqrt{2 \pi r}}
\end{gathered}
$$

(3) 若存在固溶氢, 切应力 $\tau=\tau_{C}^{*}$ 时即发生氢致 开裂， $\tau_{C}^{*}$ 值表达式为式(6)

$$
\tau_{C}^{*}=0.328 \frac{K_{I}^{*}}{\sqrt{2 \pi r}}
$$
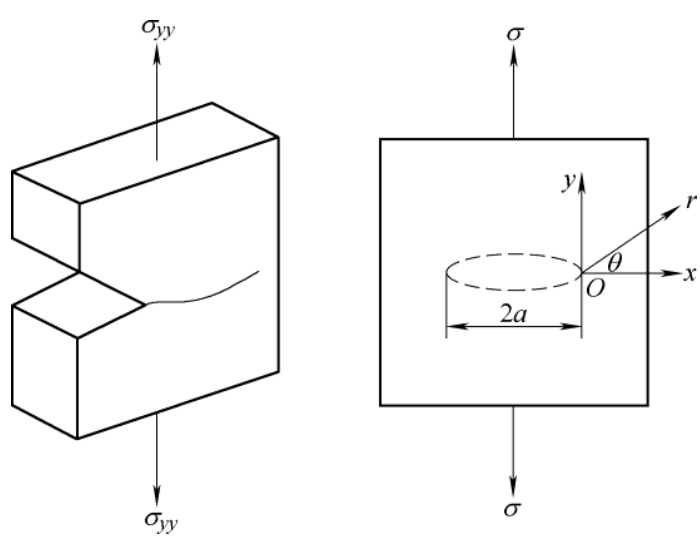

图 6 CT 试样表面裂纹扩展和局部受力示意图

充氢 $0.05 \mathrm{wt}$.\%致焊接接头表观屈服应力下降, 开裂所需应力强度因子 $K_{I}^{*}$ 较低, 局部微裂纹孕育、 萌生与扩展, 最终导致 $\mathrm{CT}$ 试样开裂, 断裂方式为 韧性断裂。此外, 富氢气团随着位错一起运动而无 法对位错运动产生 “钉扎” 作用, 氢移动至强障碍 时, 位错需要将接触面上的氢倾倒在强障碍上方才 可绕过此障碍继续运动, 这导致障碍处局部氢浓度
升高, 若超过其固溶度可析出氢化物, 进而影响 CT 试样氢致开裂过程。

\section{2 微裂纹孕育位错塞积理论}

氢化物析出明显改变位错分布和数量, 影响焊 接接头微裂纹孕育、萌生与扩展; 一方面, 氢化物 析出周围产生较大晶格畸变, 畸变能可形成新位错; 另一方面, 氢化物自身作为捕获位错的一种强陷阱, 其 “钉扎” 作用致位错运动受阻。微裂纹大多在位 错塞积群端部萌生, 金属材料位错塞积区多为晶粒边 界、晶粒内或晶界应变集中区和第二相界面位置 ${ }^{[21]}$ 。 充氢 $0.21 \mathrm{wt}$. \% 位错塞积区集中在层片 $\alpha$ 晶粒边界、 晶粒内部和氢化物析出位置。位错塞积区易产生局 部应力集中, 其中晶界处位错塞积群形成的局部应 力集中高达外应力的 $n$ 倍 ${ }^{[22]}$, 应力集中一旦达到或 超过原子键合力，微裂纹易在晶界处孕育。

晶界处析出非金属第二相时，若位错运动受阻 于第二相处, 易致微裂纹在此脆性相中孕育, 进而 诱发脆性断裂。SMITH 等 ${ }^{[20]}$ 将材料组织引入断裂过 程, 基于位错塞积和晶界上碳化物对微裂纹孕育影 响, 提出 Smith 晶界碳化物断裂模型, Smith 理论表 明: 铁素体在晶粒内部滑移或孪晶变形时产生位错 塞积, 致晶界上碳化物开裂, 微裂纹在外应力下扩 展至相邻铁素体晶粒继续扩展, 直至最终断裂。晶 界上碳化物位错塞积产生有效切应力 $\tau_{\mathrm{e}}$ 致碳化物自 身开裂条件为式(7) ${ }^{[20]}$

$$
\tau_{\mathrm{e}}=\left(\tau_{s}-\tau_{i}\right) \geqslant\left[\frac{4 E \gamma_{\mathrm{c}}}{\pi\left(1-v^{2}\right) d}\right]^{\frac{1}{2}}
$$

式中, $\tau_{\mathrm{e}}$ 为有效切应力, $\tau_{s}$ 为屈服时切应力, $\tau_{i}$ 为 位错滑移时摩擦切应力; $E$ 为弹性模量; $\gamma_{\mathrm{c}}$ 为碳化 物表面能; $v$ 为泊松比; $d$ 为晶粒直径。假定微裂纹 扩展至相邻铁素体晶粒另需能量 $\gamma_{1}$, 令铁素体有效 表面能 $\gamma_{p}=\gamma_{1}+\gamma_{\mathrm{c}}$ 得式(8) ${ }^{[20]}$

$$
\tau_{\mathrm{e}}=\left(\tau_{s}-\tau_{i}\right) \geqslant\left[\frac{4 E \gamma_{\mathrm{p}}}{\pi\left(1-v^{2}\right) d}\right]^{\frac{1}{2}}
$$

位错塞积作用下, SMITH 继续推导脆性相上微 裂纹成核 Griffith 型方程式, 构建出断裂应力 $\sigma_{f}$ 与 晶粒直径 $d$ 、碳化物厚度 $C_{0}$ 等组织参数之间的关系 特征, 获得微裂纹扩展控制断裂判据式(9) ${ }^{[20]}$

$$
\left(\frac{C_{0}}{d}\right) \sigma_{f}^{2}+\tau_{\mathrm{e}}^{2}\left\{1+\frac{4}{\pi}\left(\frac{C_{0}}{d}\right)^{\frac{1}{2}} \frac{\tau_{\mathrm{i}}}{\tau_{\mathrm{e}}}\right\}^{2} \geqslant \frac{4 E \gamma_{\mathrm{p}}}{\pi\left(1-v^{2}\right) d}
$$

假定晶界上碳化物为平板状(脆性断裂标准无 法直接用于球状碳化物), $\tau_{\mathrm{e}}$ 与 $d$ 经验关系式为 (10), 
$k_{y}^{s}$ 为剪切屈服应力 $\tau_{y}$ 与晶粒直径 $d^{-1 / 2}$ 关系斜率, 代 入式(9)得式(11)

$$
\begin{gathered}
\tau_{s}=\tau_{i}+k_{y}^{s} d^{-\frac{1}{2}} \\
C_{0} \sigma_{f}^{2}+\left(k_{y}^{s}\right)^{2}\left\{1+\frac{4}{\pi} \sqrt{C_{0}} \frac{\tau_{\mathrm{i}}}{k_{y}^{s} d}\right\}^{2} \geqslant \frac{4 E \gamma_{\mathrm{p}}}{\pi\left(1-v^{2}\right)}
\end{gathered}
$$

断裂应力 $\sigma_{f}$ 仅由组织参数碳化物厚度 $C_{0}$ 主导, $C_{0}$ 越大, $\sigma_{f}$ 越小, 越易发生脆性断裂。式(11)中将 组织因素引入断裂过程表明晶界上碳化物对临界解 理断裂应力的影响; Smith 晶界碳化物断裂模型应 用范围一般为分析体心立方晶体为母相且析出层片 状脆性第二相的所有情况 ${ }^{[20]}$ 。若仅考虑晶界上碳化 物对微裂纹扩展的影响, 忽略式(11)中第二项, 简 化 Griffith 方程为式(12) $)^{[20]}$

$$
\sigma_{f} \geqslant\left(\frac{4 E \gamma_{\mathrm{p}}}{\pi\left(1-v^{2}\right) C_{0}}\right)^{\frac{1}{2}}
$$

图 7 为层片 $\alpha$ 内部或晶界上氢化物断裂模型。 基于位错塞积 Smith 理论, 充氢 $0.21 \mathrm{wt}$. \% 焊缝层片 $\alpha$ 内部或晶界上析出氢化物时, CT 试样具体断裂过 程如下: (1) 局部塑性变形受阻于氢化物处, 位错运 动至大尺寸氢化物无法直接切过, 在氢化物界面上 产生位错塞积, 致局部区域应力集中, 微裂纹在氢 化物界面处孕育, 微裂纹优先在氢化物析出的层片 $\alpha$ 内部扩展, 后在外应力作用下扩展至相邻一侧层 片 $\alpha$ 内继续扩展, 直至 CT 试样最终断裂; (2) 氢化 物作为软基体金属中的一种脆性硬化相, 局部应力 集中致氢化物自身发生高应变, 促使微裂纹在氢化 物内部孕育, 氢化物自身断裂产生微裂纹, 其扩展 和在氢化物界面上孕育后扩展规律基本相一致。将 脆性断裂标准应用于充氢 $0.21 \mathrm{wt}$.\% 焊缝中析出的薄 片状氢化物, 断裂应力 $\sigma_{f}$ 由氢化物厚度 $C_{0}$ 主导,

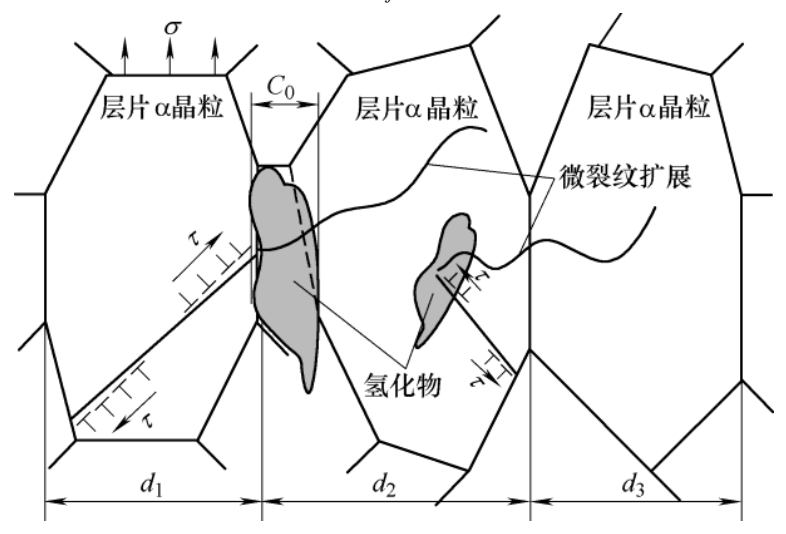

图 7 层片 $\alpha$ 内和晶界上氢化物断裂模型

$C_{0}$ 越大, $\sigma_{f}$ 越小, 氢化物越易发生脆性断裂。 $\mathrm{CT}$ 试样断裂过程裂纹尖端局部应力高度集中且一直无
法松弛, 开裂所需应力强度因子 $K_{I}$ 持续下降, 氢化 物致 $\mathrm{CT}$ 试样在更低 $K_{I}$ 下微裂纹持续孕育, 焊接接 头断裂韧度持续降低。

\section{5 结论}

（1）充氢 $0 \sim 0.21 \mathrm{wt} . \%$ 过程, CT 试样断裂韧度 $K_{Q}$ 值由 $39.6 \mathrm{MPa} \cdot \mathrm{m}^{1 / 2}$ 减小到 $22.1 \mathrm{MPa} \cdot \mathrm{m}^{1 / 2}$; 随 充氢量增加, 断裂韧度下降, 其损失最高达 $44.19 \%$ 。

(2) 随充氢量增加, 预裂区断口形貌由单一类 解理转为类解理 + 局部少量疲劳条带特征; 扩展区断 口形貌由大量韧窝+局部少量脆性断裂转为脆性断 裂特征, 充氢致焊接接头脆性断裂程度明显加剧。

(3) 应力诱导氢扩散致固溶氢局部富集形成富 氢气团, 氢气团存在内压剪切分量致产生局部塑性 变形所需外应力下降, 焊接接头表观屈服应力下降, 促进焊接接头微裂纹在较低 $K_{I}$ 下孕育、萌生和扩展; 固溶氢致焊接接头加速开裂。

（4）位错运动至大尺寸氢化物时无法直接切过, 在氢化物界面处发生位错塞积, 致局部应力集中, 微裂纹在氢化物界面处孕育; 此外, 氢化物作为软 基体金属中的一种脆性硬化相，应力集中致氢化物 发生高应变, 氢化物自身断裂, 微裂纹在氢化物内 部孕育; 氢化物致焊接接头加速开裂。

\section{参 考 文 献}

[1] 刘肖, 王理, 包陈, 等. 含轴向对称裂纹钻合金包壳管 断裂行为 [J]. 机械工程学报, 2019, 55(16): 85-90.

LIU Xiao, WANG Li, BAO Chen, et al. Fracture behaviour of zirconium alloy cladding tubes containing axial symmetric cracks[J]. Journal of Mechanical Engineering, 2019, 55(16): 85-90.

[2] SIENIAWSKI J, FILIP R, ZIAJA W. The effect of microstructure on the mechanical properties of two-phase titanium alloys[J]. Materials \& Design, 1997, 18(4-6): 361-363.

[3] 董良才, 密为建. 基于可靠性的集装箱起重机疲劳裂纹 扩展控制[J]. 中国工程机械学报, 2007, 5(4): 453-460. DONG Liangcai, MI Weijian. Reliability-based fatigue crack growth control for quay cranes[J]. Chinese Journal of Construction Machinery, 2007, 5(4): 453-460.

[4] LUETJERING G. Property optimization through microstructural control in titanium and aluminum alloys[J]. Materials Science and Engineering A, 1999, 263(2): 117-126. 
[5] 张亚军, 吕逸帆. TC4ELI 合金的断裂韧性试验研究 [J]. 材料开发与应用，2012，27(2)：14-17.

ZHANG Yajun, LÜ Yifan. Study on fracture toughness test of TC4ELI alloy[J]. Development and Application of Materials, 2012, 27(2): 14-17.

[6] PRASAD K, KAMAT S V. Dynamic fracture toughness of a near alpha titanium alloy Timetal 834[J]. Journal of Alloys \& Compounds, 2010, 491(1-2): 237-241.

[7] ROBINSON A C. Optimizing strength and fracture toughness of a cast titanium alloy through heat treatment and microstructure control[D]. Philadelphia: The Pennsylvania State University, 2007.

[8] 党宁, 赵嘉琪, 南海, 等. 退火态 ZTi6A14V 铸造钛合 金的断裂韧度研究 [J]. 航空材料学报, 2012, 32(4): 87-91.

DANG Ning, ZHAO Jiaqi, NAN Hai, et al. Fracture toughness of ZTi6Al4V casting titanium alloy under different annealed conditions[J]. Journal of Aeronautical Materials, 2012, 32(4): 87-91.

[9] KUTEPOV S M, MIKHAILIN V A. Fracture toughness of titanium alloy AT6[J]. Chemical \& Petroleum Engineering, 1992, 24(3): 254-258.

[10] 刘睿, 惠松骁, 叶文君, 等. 热处理工艺对 TB10 钛合 金动态断裂韧性的影响 $[\mathrm{J}]$. 稀有金属, 2010, 34(4): 13-18.

LIU Rui, HUI Songxiao, YE Wenjun, et al. Effects of heat-treatment on dynamic fracture toughness of TB10 titanium alloy[J]. Chinese Journal of Rare Metals, 2010, 34(4): 13-18.

[11] 张庆玲, 李兴无. TA15 钛合金两类组织对疲劳性能和 断裂韧度的影响 [J]. 材料工程, 2007(7): 4-6.

ZHANG Qingling, LI Xingwu. Effect of structure on fatigue properties and fracture toughness for TA15 titanium alloy[J]. Journal of Materials Engineering, 2007(7): 4-6.

[12] TAO B H, LI Q, ZHANG Y H, et al. Effects of post-weld heat treatment on fracture toughness of linear friction welded joint for dissimilar titanium alloys[J]. Materials Science and Engineering A, 2015，634: 141-146.

[13] POKROVSKII V V, YASNII P V, YARUSEVICH V L, et al. Study of the crack resistance of a welded joint of titanium alloy type VT6S[J]. Strength of Materials, 1988, 20(3): 316-320.

[14] SENKOV O N, FROES F H. Thermohydrogen processing of titanium alloys[J]. International Journal of Hydrogen Energy, 1999, 24(6): 565-576.

[15] 中国国家标准化管理委员会. GB/T 21143-2014 金属材 料准静态断裂韧度的统一试验方法 $[\mathrm{S}]$. 北京: 中国标 准出版社, 2015.

Standardization Administration of the People's Republic of China. GB/T 21143-2014 Metallic materials-unified method of test for determination of quasistatic fracture toughness[S]. Beijing: Standards Press of China, 2015.

[16] LIU Q M, ZHANG Z H, LIU S F, et al. The hydride precipitation mechanisms in the hydrogenated weld zone of $\mathrm{Ti}-0.3 \mathrm{Mo}-0.8 \mathrm{Ni}$ alloy argon-arc welded joints[J]. Advanced Engineering Materials, 2018, 20(5): 1700679.

[17] LIU Q M, ZHANG Z H, YANG H Y, et al. Hydride precipitation in the hydrogenated $0.12 \mathrm{wt} . \% \mathrm{H}$ weld zone of Ti-0.3Mo-0.8Ni alloy argon-arc-welded joints[J]. JOM, 2018, 70(9): 1902-1907.

[18］褚武扬, 乔利杰, 李金许, 等. 氢脆和应力腐蚀-基础 部分 $[\mathrm{M}]$. 北京: 科学出版社, 2013.

CHU Wuyang, QIAO Lijie, LI Jinxu, et al. Hydrogen embrittlement and stress corrosion cracking-basic part[M]. Beijing: Science Press, 2013.

[19] 褚武扬. 氢损伤和滞后断裂[M]. 北京: 冶金工业出版 社, 1988.

CHU Wuyang. Hydrogen damage and delayed fracture[M]. Beijing: Metallurgical Industry Press, 1988.

[20] 钟群鹏, 赵子华. 断口学 $[\mathrm{M}]$. 北京: 高等教育出版社, 2006.

ZHONG Qunpeng, ZHAO Zihua. Fractography[M]. Beijing: Higher Education Press, 2006.

[21] 涂铭旌, 滒文涁. 低合金钢低温脆性断裂论文集 [M]. 西安: 西安交通大学出版社, 1985.

TU Mingjing, YAN Wenbin. Low temperature brittle fracture of low alloy steel[M]. Xi'an: Xi'an Jiaotong University Press, 1985.

[22] WEERTMAN J, KEER L M. Dislocation based fracture mechanics[J]. Journal of Applied Mechanics, 1997, 64(4): 1029.

作者简介: 刘全明, 男, 1988 年出生, 博士后。主要研究方向为焊接结 构失效分析、钛基钎料研发及应用。

E-mail: liuquanming1988@126.com

龙伟民(通信作者), 男, 1966 年出生, 研究员, 博士研究生导师。主要 研究方向为钎焊材料、钎焊工艺与设备、钎涂熔敷技术、焊接结构失效 分析。

E-mail: brazelong@163.com 